\title{
Research on the indirect thermal drying sludge technology based on spiral equipment
}

\author{
Jian Hua Tao ${ }^{1, a}$, Bin Chen ${ }^{1, b}$, You Wen Bao ${ }^{1, c}$, Cheng Pu Jin ${ }^{1, d}$ \\ ${ }^{1}$ school of Mechanical and Electronic Engineering, Guangzhou University, Guangzhou 510006, China \\ jhtao@gzhu.edu.cnª, chaninv@163.com ${ }^{\text {b }}$,bao_youwen@163.comc, chengpuking@163.com ${ }^{\mathrm{d}}$
}

Keywords: Spiral; Sludge; Drying; Indirect

\begin{abstract}
This paper focuses on the indirect thermal drying sludge technology based on spiral equipment. At first, the paper analyzes the current situation of the sludge treatment research, and the problems caused by lacking of effective sludge treatment, including environmental pollution, ecological destruction, the dangers of people's normal lives and so on. This paper also introduces the development status of international sludge drying devices and China's domestic sludge drying processing industry. At third part of this paper introduces the principle of spiral indirect drying technology and detailed design method. At last, the indirect spiral sludge heat drying equipment which suits China's national conditions, the highly drying efficiency, easy to adjustment, free from pollution to the environment, concise and easy to maintain is designed out.
\end{abstract}

\section{Introduction}

The world organization for the water environment defines sewage sludge as "biological solid", the purpose is to show the use value of sewage sludge, U.S. EPA defines sludge as: Produced in the process of sewage disposal of solid, semi-solid or liquid residue [2]. In China, the academic on sludge has not been unified to define the sludge, which result to the sludge concept is not clear.

According to the relevant departments of statistics, the sludge was output up to 22 million tons in 2012 , and about $80 \%$ of the sludge was not effective processed. The problem of sludge treatment gets more and more attention from academies and government, and becomes a great importance environment in China. At present, the methods of urban sludge treatment in China as the following: sludge farming accounts for about $44.8 \%$, the landfill accounted for about $31 \%$, other methods about $24.7 \%$.[7,8] Landfill is not fundamentally solve the problem of sludge, and it can produce a large number of harmful gas in the course of the interpretation. If it not well seepage control measures to the harmful material in sludge, which is easy to cause the pollution of groundwater. At the same time, sludge landfill needs a large area of space and cost a lot at transportation. Looking from the angle of material circulation, sludge landfill only slow down the process of pollution to a certain extent.[8] When sludge used in agricultural, if sludge just through simple processing is directly used for crop fertilizer, harmful substances such as pathogenic microorganisms and heavy metals is not easy to clear, it is easy to enters the body through the food chain, which will detrimental to human health. In recent years, the sludge industry has got a certain development, sludge treatment development towards to diversification and harmless way, but sludge reuse become the biggest technical bottleneck in the sludge drying, and how to reduce the energy consumption problem in the process of sludge drying is the most key factors. Therefore, designing a set of complete sludge drying equipment conforms to China's national conditions and development needs will be certainly benefit to Chinese sewage sludge treatment development and the resources recycling industry development.

\section{The current situation of the development of sludge drying equipment}

Sludge drying technologies mainly include gravity concentration, mechanical dehydration, heat drying technology, and biological drying technology, etc. And the most used is thermal drying technology at home and abroad. Drying technology is the thermal medium in contact with the sludge, for evaporation heat transfer water to achieve the effect of sludge drying [3]. The main 
characteristic as follows: the sludge volume reduced obviously, which can be reduced about $1 / 5-$ 1/3; After drying, the sludge will form granular or powder products, which are more stability, and sludge performance can be improved; The drying product without excitant gas and pathogens, which can reduce the sludge on the negative effect, the sludge after processing will be secondary treated more likely. Because of the sludge thermal drying includes these advantages, the sludge heat drying technique in the sludge treatment plays more and more important role. Especially since the 1990 s, the thermal drying technology on the treatment of urban sewage sludge technology has been developed rapidly. [7]According to deference of the medium and the contact way, the sludge thermal drying technology can be divided into three categories[6,7,8,10]: direct drying method, indirect drying method and direct - indirect combined drying method, as is shown in figure 1. After the drying process, water content of the sludge can even be lower than $10 \%$, the drying rate is high, the sludge can be used as building materials company to do the raw materials of cement or tiles, and the heavy metals and all kinds of pathogens in sewage sludge can minimizes environmental pollution in the brick, and it is harmless to people. [3]

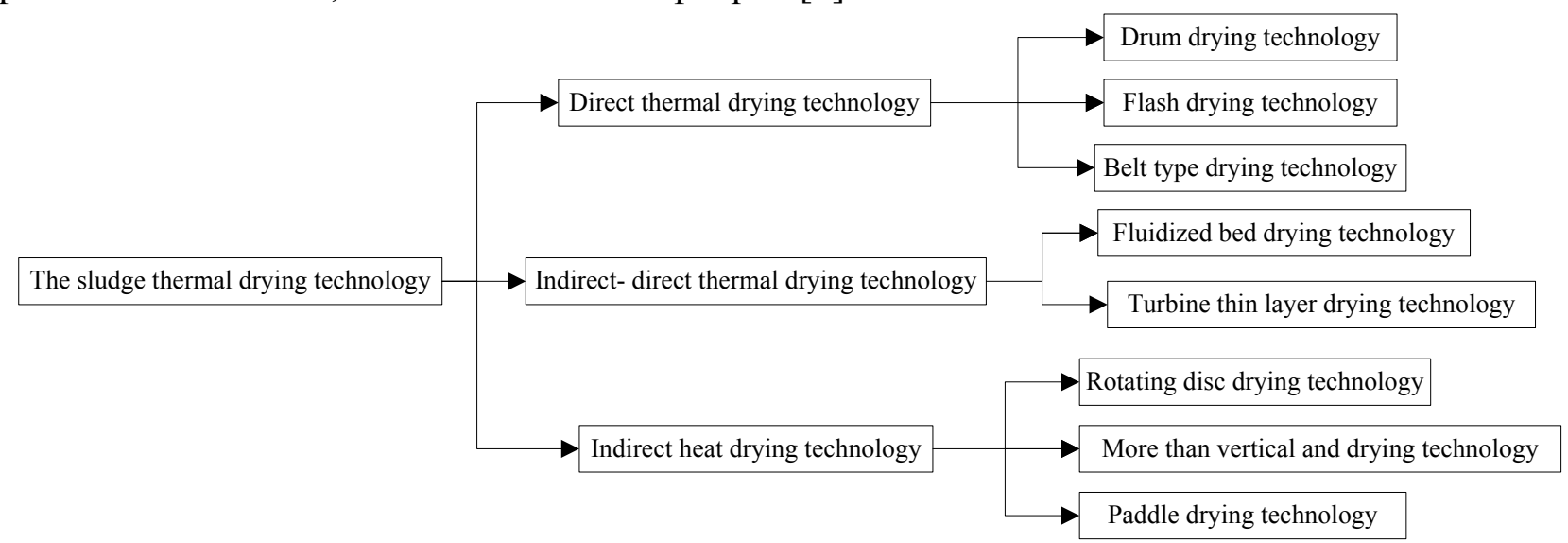

Fig.1 The main thermal drying technology existing at present

At present, the sludge drying equipments of China are mainly on the introduction of foreign technology. But there are some limitations, such as the cost is too high at repairing and maintenance of the equipment, the equipment covers a big area and poor mobility in sludge treatment process, the energy consumption is serious, these aspects are limited sludge treatment industry development in China. [7,9]

With the research to parts of the sludge production in China and the demand of sludge drying equipment, and according to the requirements of factories, spiral sludge drying equipment is designed. The design conforms to China's national conditions and it will be certainly to promote the development of industry of sludge drying in China.

\section{The working principle of spiral sludge drying equipment}

Spiral indirect sludge drying equipment is based on the screw axis as the main drying equipment, which takes the method that screw axis heats the sludge, and make the heat between sludge and screw axis has good transfer effect. The rotation of the screw axis can transport of sludge, at the same time, it also have the function of mixing sludge, which is benefit to the sludge drying. Spiral of sludge drying equipment work consists of two processes: preheating stage, sludge drying stage. Preheat phase is the process of sludge temperature rise slowly, this stage does not produce too much water vapor, but it prepare for the next stage of sludge. In this stage, the sludge's temperature is low relative to the drying equipment body temperature. The second stage is sludge drying stage, this stage is mainly in the main body of spiral sludge drying equipment, through the high temperature heating and mixing of the sludge, and makes the sludge drying at a high rate of speed.

The specific sludge drying process: the main job of sludge collection device is sludge collection, and ensure that there is always enough sludge for the sludge conveyor equipment transport; The transport equipment mainly job is to transport sludge, in the sludge transporting process, ensuring the transport sludge volume remains the same at any times; Preheating equipment's function is to 
make the sludge have the right temperature before the formal heating; The main drying body is the center of the sludge drying, sludge achieve appropriate water content and back out in it; Medium heating equipment is mainly function is to heat the transfer medium; Waste gas treatment equipment mainly function is to collect drying advocate the casing produce harmful gases, and it won't produce secondary pollution to the environment, the relationship of each part is shown in figure 2 .

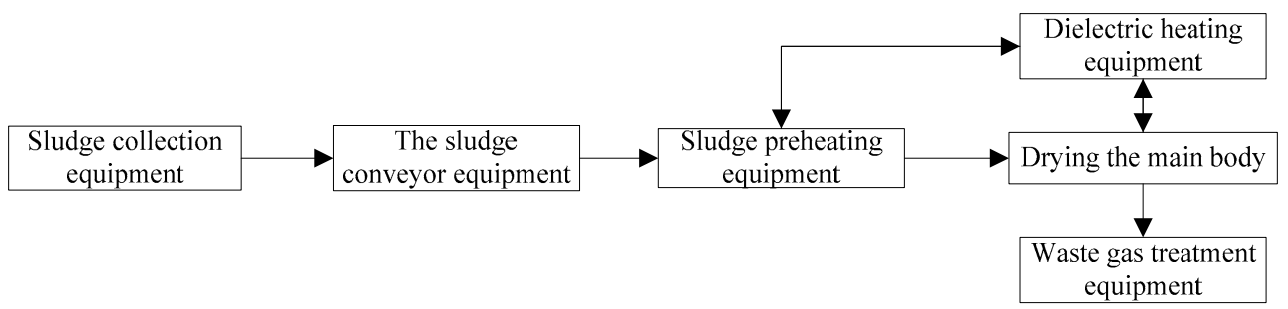

Fig.2 The main parts of the spiral of sludge drying equipment

\section{The design of the spiral sludge indirect heat drying equipment}

\subsection{The idea to achieve main function}

(1) Sludge transportation stability: Sewage treatment plants produce of sludge moisture content over $80 \%$, the sludge contains sticky smelly features, and easy to agglomerate, adhesion, if it takes ordinary belt conveyor, the stability of the sludge transportation will be difficult to guarantee. The project selects that stable screw conveyor and belt conveyor with the delivery method to make transmission relatively as a way of delivery.

(2) According to specific drying needs to set the equipment layout: The whole equipment is used the parameterized design method. At the same time, according to the drying of the specific requirements, the factory can set the specific installation arrangement and drying the number of main body, to make sure the reasonable use of the resource.

(3) Equipment is easy to control: The whole equipment motors control with two control methods: automatic control and manual control, it also can be adjusted the speed on every single motor, so as to meet the needs of actual transport.

(4) Sludge drying process is easy to monitor: Different locations in the device, sensors are installed to test sludge water content and the equipment operation. To make sure that when problems arise, it will be automatic protection, avoiding the damage to the whole equipment.

3.2 The main design idea of the indirect thermal drying sludge equipment

(1) The establishment of a heat transfer model

In the designing process of the indirect spiral heat drying sludge equipment, the establishment of a heat transfer model is the center of the whole equipment, and how to make heat medium's heat maximum passed to sludge is the main consideration. Spiral sludge drying equipment is mainly to use the indirect heat transfer medium heat for spiral propulsion unit, spiral propulsion unit with a good contact with the sludge in the main body, transfer heat to the sludge, at the same time, the spiral propulsion unit stirring effect to make the sludge moisture evaporation fast to promote drying. The main body part's diagram of the spiral indirect thermal drying sludge equipment is shown in figure 3.

After heat transfer medium flows in the heating screw propulsion unit, the hot oil transfers heat to the sludge to the greatest extent. Because of the backflow hot oil also has the very high temperature, in order to better use of heat, it can return the hot oil into the equipment dry the oil chamber of themain body directly to raise the temperature of the whole body, to provide high temperature environment for sludge drying stage. In the sludge drying process, the equipment will produce a large number of high temperature steam. In order to avoid the waste of energy, before steam into the waste gas processing plant, making the waste gas utilization, the waste gas can enter the main body of the preheating equipment to make the sludge preheated, so it can make the resources using reasonable, the main energy flow ways as shown in figure 4. 


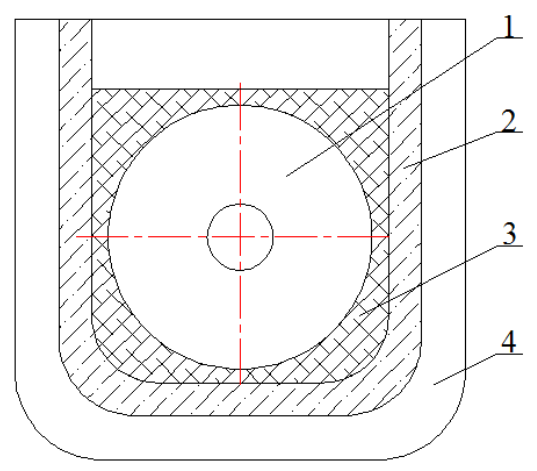

1 - high temperature spiral propulsion unit; 2 - the main body and movements of the heat exchange cavity; 3 - sludge; 4 - vacuum insulation

Fig.3 Spiral sludge drying cabinet schematic diagram

(2) The choice of sludge transportation method

Sludge transportation mode has two methods can be used: belt conveyor and screw conveyor. The conveyor belt conveyor design relatively simple, and it smooth delivery, considering the characteristics of the sludge, if only using separate belt conveyor, the biggest problem is that to ensure the stability of the sludge transportation. On this issue, the screw conveying get very good supplement, In order to facilitate processing and manufacturing, in the design of each section connection, using the same connection method, it will be greatly shorten the processing time.

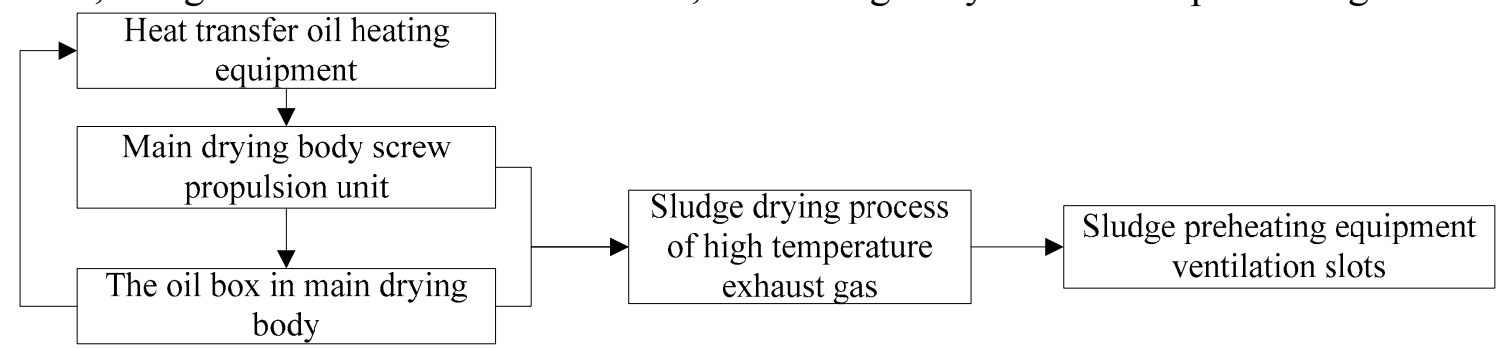

Fig.4 The energy flow in spiral sludge indirect drying equipment

Sludge preheating equipment is one part of the sludge conveyor equipment, because the sludge preheating equipment besides conveying sludge, it also preheats of the sludge to be prepared for the sludge drying stage. Therefore, sludge preheating device's transmission part is the same as in front of the screw conveyor design, it mainly in the ministry points added high temperature exhaust gas flow layer, the specific arrangement is shown in figure 5 .

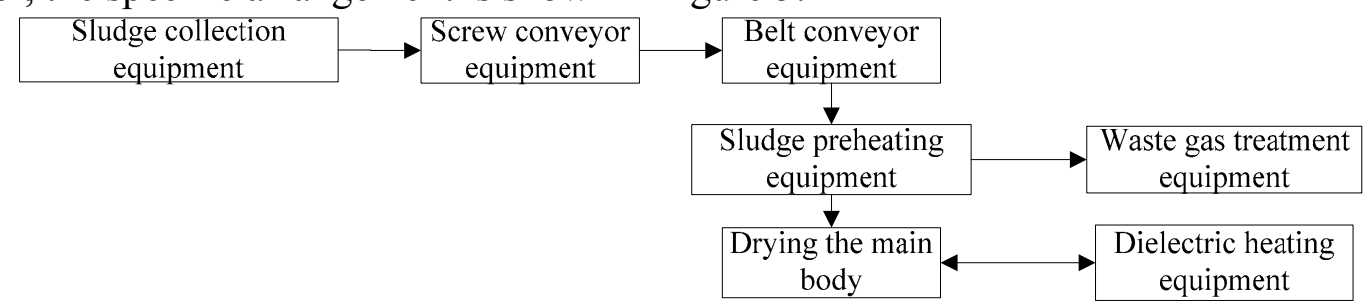

Fig.5 Spiral indirect sludge thermal drying equipment arrangement

(3) Control scheme is introduced

Spiral indirect sludge thermal drying equipment takes control of intelligent automatic control calculation method in the arrangement. According to the sensors' signal, it can automatic detection of sludge water content, according to the requirements of the final moisture content, the computer automatically calculate the central axis of the casing running speed and the main body in unit time into the mud. At the same time, in order to facilitate the user to use, the equipment can also be manually controlled, that is every one device monitor can be arbitrary rotation speed.

\section{Summary}

At present, China's sludge treatment industry has been on the right track, but sludge treatment is still in its infancy. Because the sludge is not properly handling, environment pollution and resource 
waste problem caused more and more attention. This paper is mainly about the process of sludge treatment in China currently, investigation and analysis of the requirement demand of sludge disposal, according to domestic market demand, analysis of the existing sludge drying model, to find the path of the sludge drying. And it describes the working principle of indirect spiral thermal drying sludge equipment in detail, the equipment material handling stability and flexible arrangement, the equipment is easy to control and operation process of the whole monitoring etc, and describes the design process of the device. Indirect spiral heat drying sludge equipment research and development will promote the development of sludge disposal industry in China.

\section{References}

[1]Yu Jie, Tian Ningning, Wang Kaijun, Ren Yuan, Analysis and discussion of sludge disposal and treatment of sewage treatment plants in China[J]. Chinese Journal of Environmental Engineering, $2007.1,: 82-86$.

[2]Alexandria Water Environment Federation. Biosolids Composting: A Special Publication. Water Environment Federation., 1995

[3]LI Run-dong, ZHANG Wan-li, SUN Yang, HE Ye-guang, Adaptability analysis and future development trend of sludge thermal drying technology[D], Renewable Energy Resources

[4] ZHANG Hong. Guangzhou sludge disposal and analysis of the status quo of the application[J].GuangDong: Guangdong institute of architectural design.2013.88-89

[5] Urban sewage treatment plant sludge disposal and pollution prevention and control technology policy (trial), 2010.

[6] LI Jia-xiang, HE Yang, FAN Yue-hua. Comparison \& Prospect of Four Sludge Drying Technologies \& Equipments[J]. China Municipal Engineering. 2013.2(164):80-84.

[7] WANG Gang. Present status of Treatment and Disposal Techniques of Sludge at Home And Aboard, Environmental Engineering,,2013, 31:530-534.

[8]XUE Bai. Conditioning and dewatering of sludge drying and making experiments[D]. Guangzhou: Guangzhou University.2013.5

[9] WANG Ping, HUANG Ou. The sludge thermal drying process design key issues discussed in this paper [J]. water supply and drainage, 2012, 38(5):23-27.

[10]LI Bin-bin, FAN Hai-hong, Yang AI-qing, etc. The sludge drying and gas releasing characteristics research [J]. Silicate report, 2013.2, 32(2):205-209.

[11] WANG Rui. Sludge drying characteristics and process research[D]. Shenyang: Shenyang Institute of Aeronautical Engineering, 2010.

[12] YUAN Dong-cheng, HUANG Ya-ji, SUN Yu, etc. Wet sludge in fluidized bed drying characteristics [J].Chinese Journal of Environmental Engineering , 2013.6, 7(6):2317-2321. 